05

\title{
Тепловая стабилизация \\ резистивных состояний \\ сверхпроводящих композитов: квазилинейное приближение
}

\author{
(C) В.P. Романовский \\ Национальный исследовательский центр „Курчатовский институт“, \\ 123182 Москва, Россия \\ e-mail: romanovskii@aol.com
}

(Поступило в Редакцию 21 июня 2016 г.)

Исследованы условия возникновения и развития тепловых неустойчивостей в композитном сверхпроводнике с непрерывно нарастающей вольт-амперной характеристикой, описанной степенным уравнением. Анализ условий тепловой стабилизации выполнен в обобщенном виде, используя безразмерные переменные, сохраняющие свою инвариантность при их варьировании. Для локального температурного возмущения рассчитаны критические энергии и скорости его необратимого распространения. Доказано, что у композитных сверхпроводников возможны стабильные состояния, когда предельно допустимые токи могут быть выше или ниже условно заданного значения критического тока композита. При этом разрушение сверхпроводимости при закритических токах происходит не в виде скачкообразного перехода из сверхпроводящего состояния в нормальное, а в силу формирования тепловой и электрической волн переключения, распространяющихся вдоль композитного сверхпроводника с постоянной скоростью. Для сверхпроводящих композитов со степенной вольт-амперной характеристикой сформулировано условие полной тепловой стабилизации. Результаты выполненных численных экспериментов доказывают, что существующая теория тепловой стабилизации, в рамках которой предполагается скачкообразный переход из сверхпроводящего состояния в нормальное, приводит к значительному ограничению диапазона стабильных токов, при которых сверхпроводящее состояние может сохраняться.

DOI: 10.21883/JTF.2017.04.44314.1944

\section{Введение}

Исследование условий тепловой стабилизации технических сверхпроводников является одной из основных задач, возникающих при разработке токонесущих элементов сверхпроводящих магнитных систем. Решение возникающих при этом проблем основано на анализе тепловых процессов, происходящих в сверхпроводящих композитах [1-3]. Это связано с тем, что устойчивость их сверхпроводящего состояния ограничивается в первую очередь изменением температуры композита, под которую подстраивается электрическое поле, индуцируемое в сверхпроводнике и матрице вводимым транспортным током или действием какого-либо внешнего возмущения. В рамках данного приближения были изучены основные закономерности возникновения и развития тепловых неустойчивостей (см., например, [1-11]), в низкотемпературных и высокотемпературных композитных сверхпроводниках. При этом основные положения существующей теории тепловой стабилизации были сформулированы в рамках модели, предполагающей скачкообразный переход из сверхпроводящего состояния в нормальное $(S-N$-переход). В этом случае вольт-амперная характеристика (BAX) сверхпроводника не является непрерывной функцией. В результате выделение джоулева тепла в композитном сверхпроводнике начинается только тогда, когда его температура превысит так называемую температуру резистивного перехода $T_{c s}$, при которой транспортный ток равен критическому току сверхпроводника. После этого ток начинает делиться между сверхпроводником и матрицей, а после достижения критической температуры сверхпроводника $T_{c B}$ он течет только по матрице. В то же время композитные сверхпроводники как низкотемпературные, так и высокотемпературные, в силу многих причин, например крипа магнитного потока, имеют непрерывно нарастающую ВАХ. Учет реальной формы ВАХ позволяет расширить границы общепринятого описания условий тепловой стабилизации сверхпроводящих композитов [10]. Однако сравнительный анализ условий тепловой стабилизации сверхпроводящих композитов со скачкообразной и реальной ВАХ, формулировка критериев полной стабильности сверхпроводящего состояния по отношению к тепловым возмущениям для композитных сверхпроводников с непрерывно нарастающей BAX не были выполнены. В связи с этим в настоящей работе представлены результаты исследования условий сохранения и разрушения сверхпроводящего состояния композитного сверхпроводника со степенной ВАХ при действии локального температурного возмущения. Они позволили сформулировать практически важные выводы об особенностях тепловой стабилизации сверхпроводящих композитов с реальными ВАХ как на основе низкотемпературных, так и высокотемпературных сверхпроводников. 


\section{Основные уравнения}

Рассмотрим термически тонкий сверхпроводящий композит $(-l<x<l)$ с площадью поперечного сечения $S$, несущий транспортный ток с плотностью $J$ и помещенный в постоянное внешнее магнитное поле $B$. Пусть в начальный момент времени локальная часть композита $-x_{1}<x<x_{1}$, охлажденного до температуры хладагента $T_{0}$, мгновенно нагрелась до температуры $T_{1}$. Будем считать, что распределение температуры $T$ и напряженности электрического поля $E$ по сечению композита равномерно; между сверхпроводником и матрицей имеется идеальный тепловой и электрический контакт; объемная доля сверхпроводника в композите равна $\eta$; ВАХ сверхпроводника описывается степенным уравнением $E(J)=E_{c}\left[J / J_{c}(T, B)\right]^{n}$, где $J_{c}(T, B)-$ критическая плотность тока сверхпроводника, определенная при априори заданном значении напряженности электрического поля $E_{c}, n-$ параметр нарастания BAX; изменение $J_{c}(T, B)$ с температурой опишем линейной зависимостью вида [1-3]

$$
J_{c}(T, B)=J_{c 0}(B)\left(T_{c B}(B)-T\right) /\left(T_{c B}(B)-T_{0}\right),
$$

где $J_{c 0}(B)$ и $T_{c B}(B)-$ критические значения плотности тока сверхпроводника и температуры в заданном внешнем магнитном поле соответственно.

Поместим начало координат в центр участка начального возмущения, и запишем уравнение распространения тепла вдоль композита в виде

$$
C \frac{\partial T}{\partial t}=\lambda \frac{\partial^{2} T}{\partial x^{2}}-\frac{h p}{S}\left(T-T_{0}\right)+\left\{\begin{array}{l}
G_{c s} \\
G
\end{array}\right.
$$

с начально-краевыми условиями

$$
\begin{gathered}
T(x, 0)= \begin{cases}T_{1}=\text { const }, & |x| \leq x_{1} \\
T_{0}=\text { const }, & x_{1}<|x|<l\end{cases} \\
\frac{\partial T}{\partial x}(0, t)=0, \quad T(l, t)=T_{0},
\end{gathered}
$$

учитывающими симметричное распределение температуры по длине композита. Здесь $C$ и $\lambda-$ объемная теплоемкость и коэффициент теплопроводности композита соответственно, $h-$ коэффициент теплоотдачи, $p-$ охлаждаемый периметр, $x_{1}$ - полудлина начального участка возмущения, $l-$ общая полудлина композита $\left(l \gg x_{1}\right), G_{c s}$ и $G-$ тепловыделения в композите, рассчитываемые либо в рамках модели скачкообразной $\mathrm{BAX}$, либо с учетом ее непрерывного нарастания соответственно.

При скачкообразном переходе из сверхпроводящего состояния в нормальное величина $G_{c s}=E J$ записыва- ется в общепринятом виде [1-3]

$$
\begin{aligned}
& G_{c s}(T)=\frac{J^{2} \rho_{n}(T, B)}{1-\eta} \\
& \times \begin{cases}1, & T>T_{c B}, \\
\left(T-T_{c s}\right) /\left(T_{c B}-T_{c s}\right), & T_{c s} \leq T \leq T_{c B}, \\
0, & T<T_{c s}=T_{c B}- \\
& -\left(T_{c B}-T_{0}\right) J /\left(\eta J_{c 0}\right) .\end{cases}
\end{aligned}
$$

В случае непрерывной ВАХ для расчета джоулевых потерь $G=E J$ воспользуемся моделью параллельного соединения двух проводящих сред. Тогда мгновенные значения напряженности электрического поля и плотностей токов удовлетворяют системе уравнений

$$
\begin{gathered}
E=E_{c}\left[J_{s c} / J_{c}(T, B)\right]^{n}=J_{m} \rho_{m}(T, B), \\
J=\eta J_{s c}+(1-\eta) J_{m} .
\end{gathered}
$$

Здесь $J_{s c}$ и $J_{m}-$ плотности токов, протекающих в сверхпроводящей части композита и в матрице соответственно, $\rho_{m}-$ удельное электрическое сопротивление матрицы.

Из системы (5) в соответствии с равенством (1) нетрудно найти выражение

$$
G(T)= \begin{cases}\eta J_{c 0} E\left(1-\frac{T-T_{0}}{T_{c B}-T_{0}}\right)\left(\frac{E}{E_{c}}\right)^{1 / n}+(1-\eta) \frac{E^{2}}{\rho_{m}}, & T<T_{c B}, \\ (1-\eta) \frac{E^{2}}{\rho_{m}}, & T \geq T_{c B},\end{cases}
$$

описывающее тепловыделение в сверхпроводящем композите со степенным уравнением ВАХ сверхпроводника.

Выполним квазилинейный анализ тепловых процессов в сверхпроводящем композите, предполагая, что значения $C, \lambda, h$ и $\rho_{m}$ не зависят от температуры. В этом случае от температуры зависят только значения $G_{c s}$ и $G$. Введем безразмерные переменные

$$
\begin{gathered}
i=J /\left(\eta J_{c 0}\right), \quad e=E / E_{c}, \quad \theta=\left(T-T_{0}\right) /\left(T_{c B}-T_{0}\right), \\
g_{c s}=G_{c s} /\left(\eta J_{c 0} E_{c}\right), \quad g=G /\left(\eta J_{c 0} E_{c}\right), \\
\varepsilon_{1}=E_{1} / E_{c}, \quad X=x / L_{x}, \quad \tau=t / t_{x},
\end{gathered}
$$

где

$$
\begin{gathered}
E_{1}=\eta J_{c 0} \rho_{m}(1-\eta), \quad L_{x}=\left(\frac{\lambda\left(T_{c B}-T_{0}\right) S}{E_{c} I_{c 0}}\right)^{1 / 2}, \\
t_{x}=\frac{C L_{x}^{2}}{\lambda}, \quad I_{c 0}=\eta J_{c 0} S .
\end{gathered}
$$

Тогда исходная начально-краевая задача преобразуется к виду

$$
\begin{gathered}
\frac{\partial \theta}{\partial \tau}=\frac{\partial^{2} \theta}{\partial X^{2}}-\frac{1}{\alpha_{E}} \theta \\
+\left\{\begin{array}{c}
g_{c s}(\theta)-\text { для модели скачко- } \\
\text { образного S-N-перехода, } \\
g(\theta)-\text { для модели степенной ВАХ, }
\end{array}\right.
\end{gathered}
$$




$$
\begin{aligned}
& \theta(X, 0)= \begin{cases}\theta_{1}, & |X| \leq X_{1} \\
0, & X_{1}<|X|<L,\end{cases} \\
& \frac{\partial \theta}{\partial X}(0, \tau)=0, \quad T(L, \tau)=0 .
\end{aligned}
$$

Здесь $L=l / L_{x}$,

$$
\begin{gathered}
g_{c s}(\theta)=i^{2} \varepsilon_{1} \begin{cases}1, & \theta>1, \\
(\theta-1+i) / i, & 1-i \leq \theta \leq 1, \\
0, & \theta<1-i,\end{cases} \\
g(\theta)= \begin{cases}(1-\theta) e^{1+1 / n}+e^{2} / \varepsilon_{1}, & \theta<1, \\
e^{2} / \varepsilon_{1}, & \theta \geq 1,\end{cases}
\end{gathered}
$$

$\alpha_{E}=\frac{E_{c} I_{c 0}}{h p\left(T_{c B}-T_{0}\right)}-$ параметр тепловой стабилизации для композита с непрерывно нарастающей ВАХ сверхпроводника, для описания которой используется априори заданное значение $E_{c}$.

Сформулированная задача (7) -(9) позволяет выполнить сравнительный анализ основных характеристик диссипативных состояний композитных сверхпроводников с транспортным током на основе широко используемых моделей BAX и объяснить их влияние на механизмы, лежащие в основе развития в них тепловых процессов при действии температурного возмущения конечной протяженности и различной мощности, варьируя параметр тепловой стабильности $\alpha_{E}$, безразмерный ток $i$, параметры температурного возмущения. Подчеркнем, что введенные безразмерные переменные и параметры не являются взаимозависимыми [12], и поэтому они не приводят к нарушению инвариантности задачи (7). Отметим также, что сформулированные модели не требуют подгонки исходных параметров друг к другу, так как в линейном приближении (1) критические параметры сверхпроводника имеют один и тот же физический смысл. Как следствие, в получаемых результатах должен соблюдаться предельный переход от модели со степенной ВАХ к модели скачкообразного $S-N$-перехода при $n \rightarrow \infty$.

Для того чтобы избежать влияния граничного условия при $X=L$ на характер пространственного изменения температуры композита, в численных расчетах соблюдалось условие $X_{1} \ll L=100$.

В рамках предложенных безразмерных переменных появляется параметр $\varepsilon_{1}$, который по своему смыслу описывает влияние электрического сопротивления матрицы на интенсивность процесса деления тока. Оценим его возможное значение для высокотемпературного сверхпроводника, покрытого серебром, полагая, что $\rho_{m} \sim 10^{-7} \Omega \cdot \mathrm{cm}, J_{c 0} \sim 10^{5} \mathrm{~A} / \mathrm{cm}^{2}, \eta \sim 0.5$, $T_{c B}-T_{0} \sim 20 \mathrm{~K}, E_{c} \sim 10^{-6} \mathrm{~V} / \mathrm{cm}$. Тогда $E_{1} \sim 10^{-2} \mathrm{~V} / \mathrm{cm}$ и $\varepsilon_{1} \sim 10^{4}$.

Для удобства проводимого анализа ниже вместо параметра тепловой стабилизации $\alpha_{E}$ используется известный параметр Стекли. Согласно [1-3], он равен $\quad \alpha=\frac{I_{c \circ}^{2} \rho_{m}}{h p\left(T_{c B}-T_{0}\right) S(1-\eta)}$. Поэтому имеет место связь $\alpha=\varepsilon_{1} \alpha_{E}$. Но в этом случае необходимо учитывать, что параметр Стекли оказывается зависимым от безразмерного параметра $\varepsilon_{1}$, в то время как параметр тепловой стабилизации $\alpha_{E}$ от него не зависит.

\section{Особенности формирования и разрушения стабильных состояний композитного сверхпроводника}

На рис. 1 изображены характерные температурные зависимости мощности тепловыделения в композите и теплового потока с его поверхности. На вставке некоторые из них показаны более детально. Расчет проводился при $n=25, \alpha=1, \varepsilon_{1}=10^{4}$ и различных токах (как докритических, так и закритических). Сплошные линии соответствуют значениям $g(\theta)$, вычисленным согласно (9). Штриховыми линиями обозначены тепловыделения $g_{c s}(\theta)$, которые описываются выражением (8) и были рассчитаны при $i=0.8$ и $i=1.095$. Штрих-пунктирная линия показывает тепловой поток в хладагент $\left(w=\varepsilon_{1} \theta / \alpha\right)$. Приведенные диаграммы состояний наглядно демонстрируют характерные качественные особенности формирования устойчивых и неустойчивых состояний сверхпроводящих композитов.

Режимы 1 и 2 имеют только одно равновесное состояние при температуре $\theta_{i}$, при которой кривые $g(\theta)$ и $w(\theta)$ пересекаются. Это значение изображено на вставке к рис. 1 для режима 4. Уравнение для определения $\theta_{i}$ выписано ниже. Подобное равновесное состояние не возникает при моделировании тепловых режимов, когда используется скачкообразная ВАХ. Режим 2 соответствует предельному значению тока $i_{r}$, после превышения

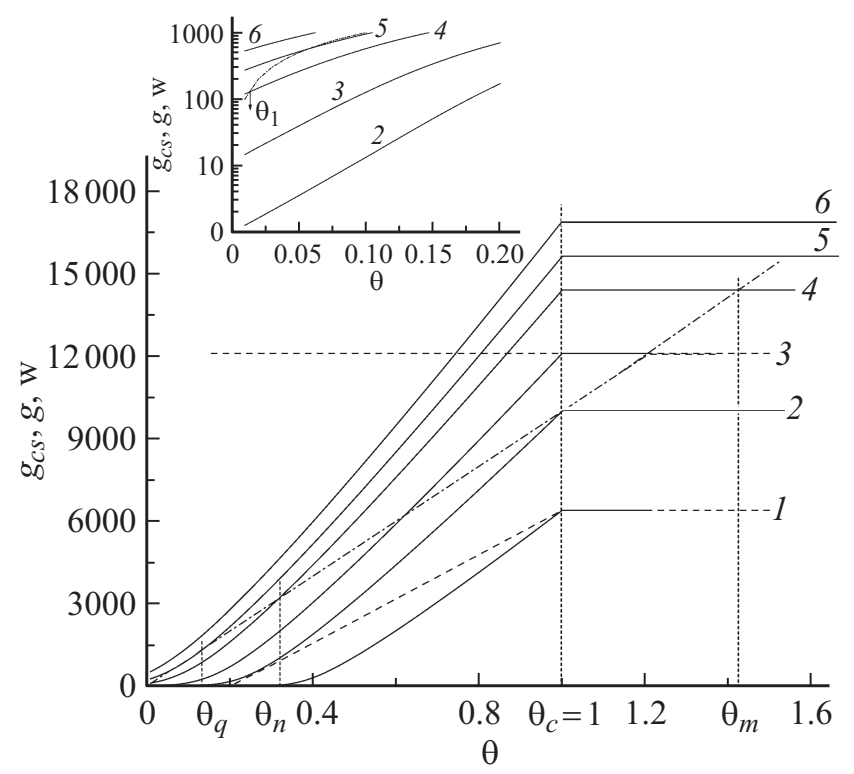

Рис. 1. Характерные диаграммы состояний композитного сверхпроводника при различных токах, $i: 1-0.8,2-1$, $3-1.095,4-1.2,5-1.255,6-1.3 ;-$ - модель со степенной ВАХ, --- — модель скачкообразного $S-N$-перехода. 


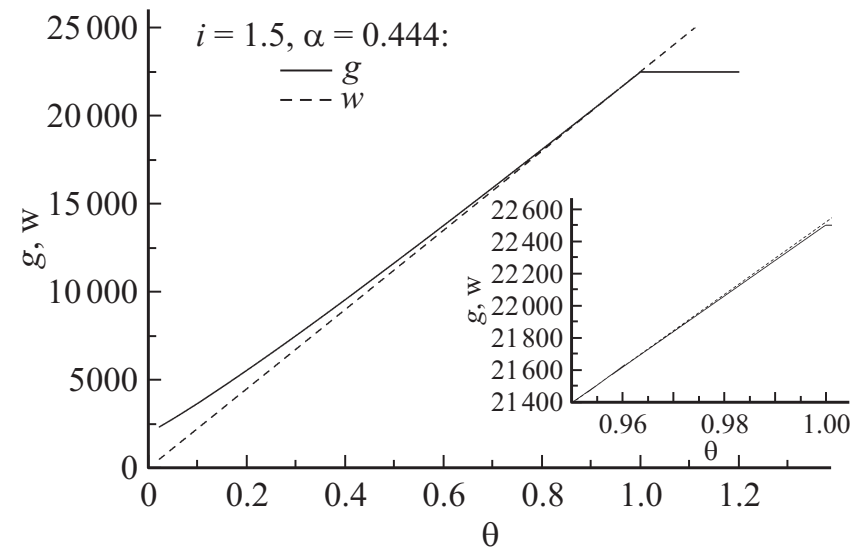

Рис. 2. Устойчивое диссипативное состояние композита при закритическом токе, не приводящим к разрушению его сверхпроводящих свойств.

которого появятся дополнительные равновесные точки. Его значение следует из очевидного равенства $\alpha i_{r}^{2}=1$. Данный токовый режим известен как минимальный ток существования нормальной зоны [1-3]. В рамках рассматриваемого приближения его величина не зависит от сформулированных выше моделей. Соответственно для всех $i<i_{r}$ конечная температура композита с реальной $\mathrm{BAX}$ после действия любого теплового возмущения по истечению некоторого времени будет равна $\theta_{i}$, так как сверхпроводящий композит при $i<i_{r}$ не переходит необратимым образом в нормальное состояние. Этот вывод справедлив и для закритических токовых режимов. В этом случае для любого заданного тока $i>1$ существует граничное значение параметра тепловой стабилизации, равное $\alpha_{r}=1 / i^{2}$, когда для всех $\alpha<\alpha_{r}$ тепловая стабильность композита, несущего закритический ток, к тепловым возмущениям с произвольной энергией сохраняется. Для данных параметров тепловой стабилизации конечная температура композита после действия произвольного теплового возмущения не будет превышать критическую температуру сверхпроводника, так как $\theta_{i}<1$. В качестве иллюстрации существования данных устойчивых закритических состояний на рис. 2 приведены $(g-w)$ диаграммы, вычисленные при $i=1.5$ и $\alpha=0.444$. На вставке к рисунку диаграммы $(g-w)$ построены более детально в диапазоне температур, близких к $\theta_{i}$. Подчеркнем, что в рамках существующей теории тепловой стабилизации подобные режимы не принимаются во внимание.

При токах, превышающих значение $i_{r}$, (токовые режимы 3 и 4), могут существовать три равновесные точки. Они изображены для режима 4 и описывают следующие состояния композита. Во-первых, в области малых перегревов также имеет место температура устойчивого равновесия $\theta_{i}$. Во-вторых, возникает максимальная температура устойчивого равновесия $\theta_{m}>1$, к которой будет стремиться композит, в частности, в результате необратимого перехода в нормальное состояние [1-3].
Так как при $\theta>1$ джоулевы потери, согласно выбранной модели, использующей линейную температурную зависимость плотности критического тока, определяются потерями только в матрице, то значение $\theta_{m}$ не зависит от сформулированных выше моделей ВАХ. Поэтому в рамках рассматриваемых приближений $\theta_{m}=\alpha i^{2}$. В-третьих, появляется температура неустойчивого равновесия $\theta_{n}$, по своему физическому смыслу соответствующая критическому значению температуры $\theta_{1}$ при действии бесконечно протяженного возмущения $\left(X_{1} \rightarrow \infty\right)$ [8]. Легко понять, что величина $\theta_{n}$ будет зависеть от интенсивности охлаждения (в общем случае от значения $\alpha$ ). В частности, в предельном случае может выполняться условие $\theta_{m}=\theta_{n}=1$. Подобное состояние изображено на рис. 3 .

Значения $\theta_{i}$ и $\theta_{n}$ следуют из очевидного равенства $w=g$. Оно приводит к трансцендентному уравнению

$$
\alpha i^{2}=\alpha i\left(\varepsilon_{1} / \alpha i\right)^{1 / n}(1-\theta) \theta^{1 / n}+\theta,
$$

которому удовлетворяют оба этих значения.

Закритический режим 3 был рассчитан для сверхпроводника со степенной ВАХ на основе известной теоремы равных площадей [1-3], которая позволяет определить так называемый минимальный ток распространения нормальной зоны $i_{s}$ (уравнение, которое использовалось для его определения, выписано ниже). Нетрудно понять, что его значение зависит от используемых моделей ВАХ в силу соответствующего отличия значений $g_{c s}(\theta)$ и $g(\theta)$. Причем заметное различие будет достигаться при интенсивном охлаждении. В диапазоне токов $i_{r}<i<i_{s}$ сверхпроводящее состояние композита устойчиво к тепловым возмущениям с произвольной энергией. Но при более высоких токах $\left(i_{s}<i\right)$ стабильность сверхпроводящего состояния начинает зависеть от характера теплового возмущения [8].

Режимы 5 и 6 имеют только одну равновесную точку $\theta_{m}$. При этом предельное токовое состояние 5 соответствует так называемому току срыва $i_{q}$ [13], после превышения которого (режим 6) сверхпроводящее состояние композита неустойчиво, даже если тепловые

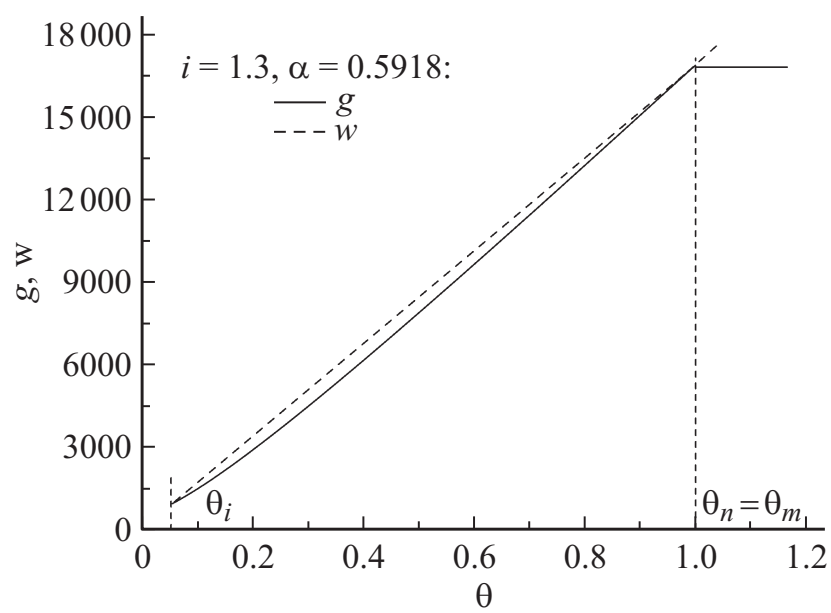

Рис. 3. Диаграмма состояний при $\theta_{m}=\theta_{n}$. 
возмущения отсутствуют, поскольку тепловыделение превышает тепловой поток в хладагент для всех $\theta<\theta_{m}$. Току $i_{q}$ соответствует конечный перегрев композита $\theta_{q}$ и напряженность электрического поля $e_{q}$. Согласно [13], и как следует из рис. 1 , параметры срыва $\theta_{q}, i_{q}$ и $e_{q}$ при отсутствии внешних тепловых возмущений можно определить из условия $w=g, d w / d \theta=d g / d \theta$ при $\theta=\theta_{q}$. Эти условия приводят к следующим выражениям:

$$
\begin{gathered}
\left(\varepsilon_{1} \theta_{q}\right)^{2}\left[\alpha \theta_{q}\left(\theta_{q}+n-1\right)\right]^{n-1}\left[(n+1) \theta_{q}-1\right]^{n+1}=\left(n \theta_{q}\right)^{2 n}, \\
i_{q}=\sqrt{\frac{n \theta_{q}\left(1-\theta_{q}\right)}{\alpha\left[(n+1) \theta_{q}-1\right]}+\frac{\theta_{q}}{\alpha}}, \quad e_{q}=\frac{\varepsilon_{1} \theta_{q}}{\alpha i_{q}}
\end{gathered}
$$

описывающим параметры режима срыва. Отметим, что в этом случае $\theta_{i}=\theta_{n}=\theta_{q}$.

Существование $\theta_{q}, i_{q}$ и $e_{q}$ показывает, что у сверхпроводящих композитов с реальной ВАХ возникновение неустойчивости вводимого тока в общем случае не происходит при условно заданном критическом токе. Согласно (11), для рассматриваемого композита $i_{q}=1.25$, что на $25 \%$ больше заданного критического тока, несмотря на конечное значение $\theta_{q}$. Более того, модель скачкообразного $S-N$-перехода не только не позволяет описать предельные токовые режимы, но и не позволяет корректно сформулировать условие полной тепловой стабилизации композитного сверхпроводника, когда произвольное начальное возмущение не будет переводить его в нормальное состояние во всем диапазоне токов вплоть до тока срыва. Запишем данное условие, используя (11) и принимая во внимание, что при граничных значениях параметра тепловой стабилизации $\alpha_{f}$ и тока $i_{f}$, при которых соблюдается условие полной тепловой стабилизации, температура композита перед возникновением неустойчивости равна критической температуре сверхпроводника $\left(\theta_{q}=1\right)$, а введенный ток будет стабильно течь только по матрице $\left(\alpha_{f} i_{f}^{2}=1\right)$. Тогда значение параметра полной тепловой стабилизации следует из равенства

$$
\alpha_{f}^{n-1} \varepsilon_{1}^{2}=1
$$

При этом предельное значение тока, равное

$$
i_{f}=\varepsilon_{1}^{1 /(n-1)},
$$

описывает ток, после превышения которого композит будет находиться в устойчивом несверхпроводящем состоянии. В размерных переменных условие полной тепловой стабильности будет соблюдаться, если исходные параметры удовлетворяют неравенствам

$$
\begin{gathered}
\left(\frac{\eta J_{c 0}^{n}}{E_{c}}\right)^{2}\left[\frac{S}{h p\left(T_{c B}-T_{0}\right)}\right]^{n-1}\left(\frac{\rho_{m}}{1-\eta}\right)^{n+1} \leq 1, \\
J \leq \eta J_{c 0}\left[\frac{\eta J_{c 0} \rho_{n}}{(1-\eta) E_{c}}\right]^{1 /(n-1)} .
\end{gathered}
$$

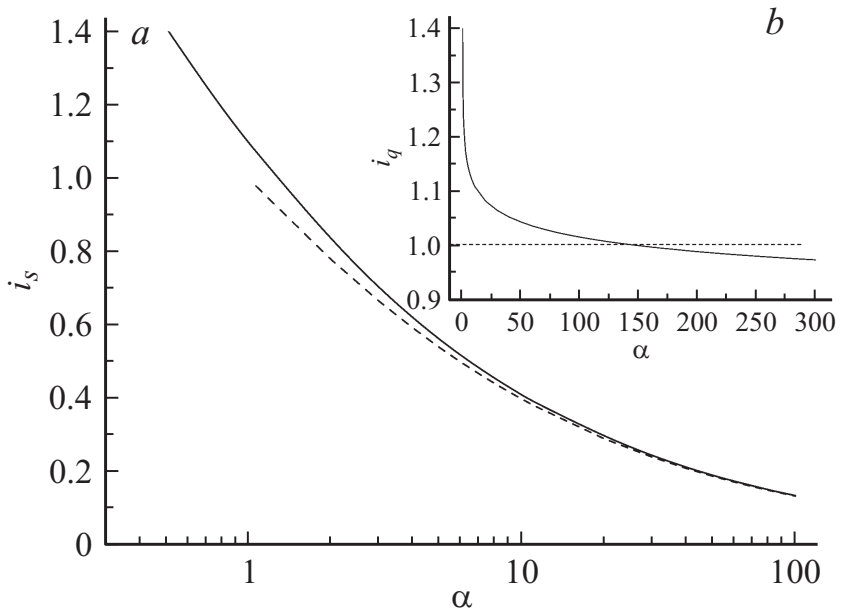

Рис. 4. Зависимость минимального тока распространения нормальной зоны $(a)$ и тока срыва $(b)$ от параметра тепловой стабилизации.

Отметим, что хотя критериальные соотношения (11) формально отличаются от соответствующих критериев возникновения токовой неустойчивости, сформулированных ранее в [14], они приводят к тем же значениям $\theta_{q}, i_{q}$ и $e_{q}$. Кроме этого, критерии $(10)-(14)$ соблюдают предельный переход к модели скачкообразного $S-N$-перехода $[1-3,8]$. Действительно, при $n \rightarrow \infty$ нетрудно найти

$$
\theta_{n}=\frac{\alpha i(1-i)}{\alpha i-1}, \quad \theta_{i}=0, \quad \alpha_{f}=1, \quad i_{f}=1 .
$$

Следовательно, только в предельном случае, существующем при скачкообразном $S-N$-переходе, сверхпроводящий композит полностью стабилизирован во всем диапазоне токов вплоть до критического при условии $\alpha_{f}=1$ (так называемом условии Стекли [1-3]). Другими словами, выражения и критерии (12)-(14) наглядно демонстрируют стабилизирующую роль дифференциального сопротивления непрерывно нарастающей ВАХ сверхпроводника в устойчивости закритических токовых режимов, когда при токах, превышающих критический ток композита $(i>1)$, он может находиться в стабильном состоянии.

Перейдем от качественного анализа условий устойчивости тепловых состояний композитного сверхпроводника к их количественному описанию. Ниже приведены результаты численного решения задачи (7)-(9) для композита с параметрами $n=25$ и $\varepsilon_{1}=10^{4}$.

На рис. 4 представлены результаты расчета минимального тока распространения нормальной зоны (рис. 4, a) и тока срыва (рис. 4,b). Сплошная линия на рис. 4, $a$ описывает значения $i_{s}$, вычисленные в рамках модели со степенной ВАХ, а штрих - по модели скачкообразного $S-N$-перехода. В первом случае минимальный ток распространения нормальной зоны определялся численно, 
согласно интегральному равенству

$$
\int_{0}^{\alpha i^{2}}\left[g(\theta)-\frac{\varepsilon_{1}}{\alpha} \theta\right] d \theta=0,
$$

которое следует из теоремы равных площадей. При скачкообразном $S-N$-переходе минимальный ток распространения нормальной зоны равен [1-3]

$$
i_{s}=(\sqrt{1+8 \alpha}-1) /(2 \alpha) \text {. }
$$

Расчет предельно допустимого тока $i_{q}$ выполнялся согласно (11). Как обсуждалось выше, значения $i_{q}$ ограничиваются сверху током полной стабилизации $i_{f}$.

Приведенные кривые показывают, что в рамках рассматриваемых моделей заметное отличие между значениями $i_{s}$, рассчитанными по обеим моделям ВАХ, будет наблюдаться для „хорошо“ стабилизированных композитов $(\alpha<10)$. Оно возрастает по мере приближения к режиму полной тепловой стабилизации. Для заданных исходных параметров значение параметра полной тепловой стабилизации равно $\alpha_{f}=0.4642$. Так как оказывается, что для композитных сверхпроводников с реальной ВАХ параметр полной тепловой стабилизации может быть меньше единицы, то в нарушении условия Стекли тепловые неустойчивости могут возникать при $\alpha<1$. В общем случае значения $i_{q}$ и $\alpha_{f}$ зависят от $\varepsilon_{1}$, т.е. от сопротивления матрицы. Данная зависимость отсутствует в модели скачкообразного $S-N$-перехода.

Штриховая линия на рис. $4, b$ соответствует безразмерному значению критического тока сверхпроводника. Видно, что токи возникновения неустойчивости, определенные как максимально устойчивые токи, вводимые в композит, могут быть как закритическими, так и докритическими. Последние будут наблюдаться у „плохо“ стабилизированных композитов, в частности, при их неинтенсивном охлаждении $(\alpha \gg 1)$. В предельном случае $\alpha \rightarrow \infty$ (теплоизолированный композит) $i_{q} \rightarrow 0$ согласно (11). Данные особенности объясняются тем, что у композитов с реальной ВАХ предельно допустимый ток является следствием теплового равновесия джоулева тепловыделения с тепловым потоком в хладагент. Поэтому саморазогрев теплоизолированного композита, строго говоря, приводит к абсолютно неустойчивому состоянию. Тем самым результаты, представленные на рис. $4, b$, строго доказывают, что для композитов даже на основе жестких сверхпроводников с сильным пиннингом понятие критический ток не имеет физического смысла.

На рис. 5 показана кинетика „нормальной зоны“ (рис. 5,a), температуры композита и напряженности электрического поля (рис. 5, $b$ ) при докритическом токе $i=0.9$ для двух характерных значений параметра тепловой стабилизации: $\alpha=2-$ „хорошо“ стабилизированный композит и $\alpha=100$ —, „плохо“ стабилизированный композит. Параметры начального температурного возмущения задавались равными $X_{1}=1$ и $\theta_{1}=1$,

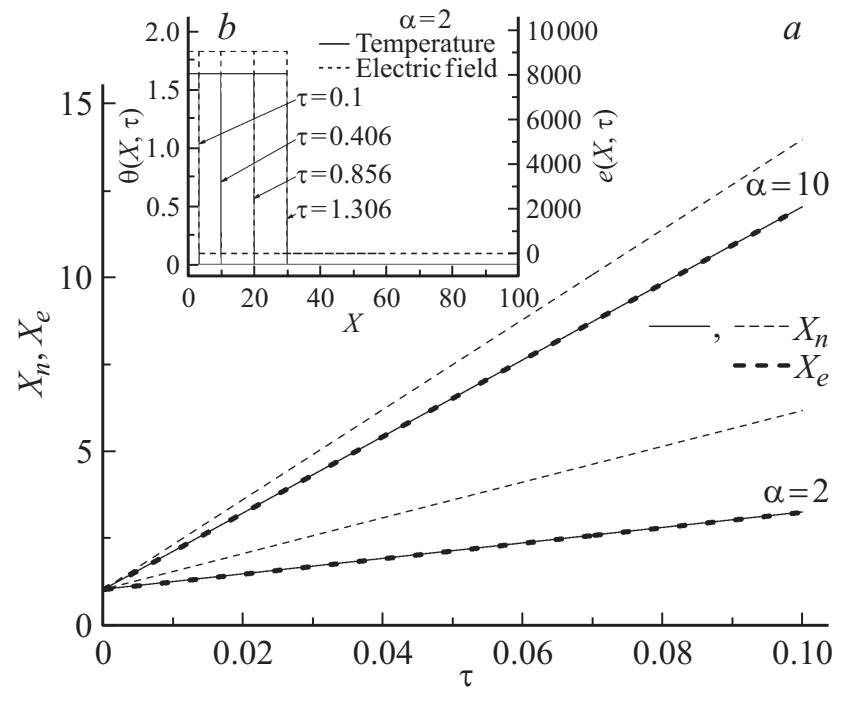

Рис. 5. Изменение во времени протяженности температурного возмущения $(a)$, температуры и напряженности электрического поля $(b)$.

которые соответствуют закритическому температурному возмущению, т.е. возмущению, которое приводит к необратимому разрушению сверхпроводимости. Штрих на рис. 5, $a$ соответствует расчетам, выполненным по модели скачкообразного $S-N$-перехода, сплошные линии и маркер „-“ - по модели со степенной ВАХ. Поскольку при моделировании тепловых состояний сверхпроводящих композитов с непрерывной ВАХ понятие нормальной зоны отсутствует в силу отсутствия границы $S-N$-перехода, то изменение ее „протяженности“ во времени определялось по кинетике точки с температурой $\theta=1-i$ (в размерном виде $T=T_{c s}$ ), а также точки с напряженностью электрического поля $e=10$. В первом случае результаты расчетов описаны сплошными линиями, во втором - маркером „-““. Видно, что при определении скорости необратимого распространения тепловой неустойчивости оба приближения приводят к эквивалентным результатам.

Из рис. 5 следует вывод о том, что после действия теплового возмущения с энергией, превышающей критическое значение, в композите формируются тепловая и электрическая волны, распространяющиеся вдоль композита с одинаковой постоянной скоростью (так называемые волны переключения [3]). Они существуют в диапазоне токов $i_{r}<i<i_{q}$ (рис. 6). При этом скорости волны переключения отрицательны при $i_{r}<i<i_{s}$ и положительны при $i_{s}<i<i_{q}$. Эти выводы справедливы и для токов, превышающих критический ток (рис. 7). В то же время, согласно рис. 1, образование теплоэлектрической волны переключения не будет происходить при $i<i_{r}$ и $i>i_{f}$. В этих случаях процессы исчезновения или необратимого распространения начального температурного возмущения носят существенно нестационарный характер в силу заметного отличия между значениями 


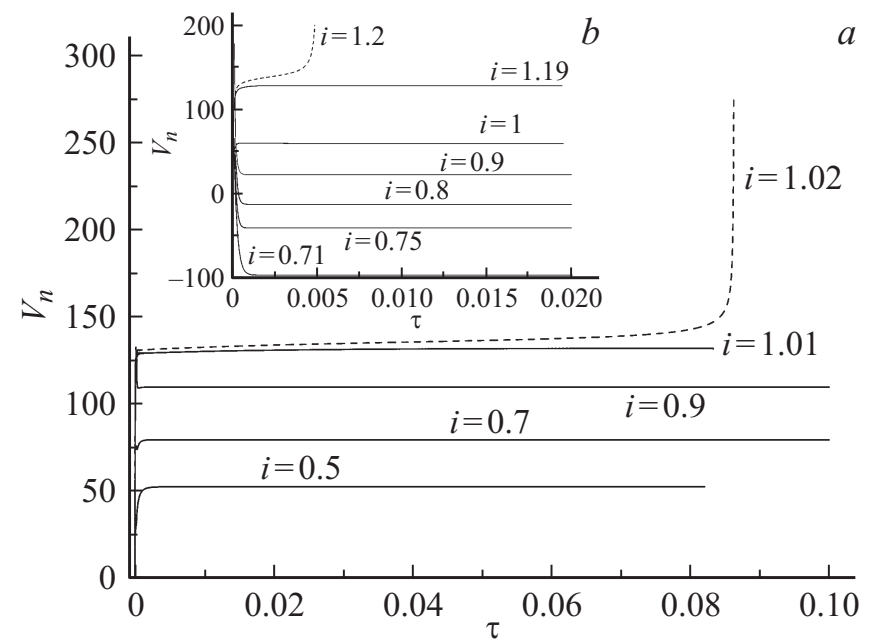

Рис. 6. Образование волны переключения при $\alpha=100(a)$ и $\alpha=2(b)$ и различных значениях тока (-- - срыв токового режима).

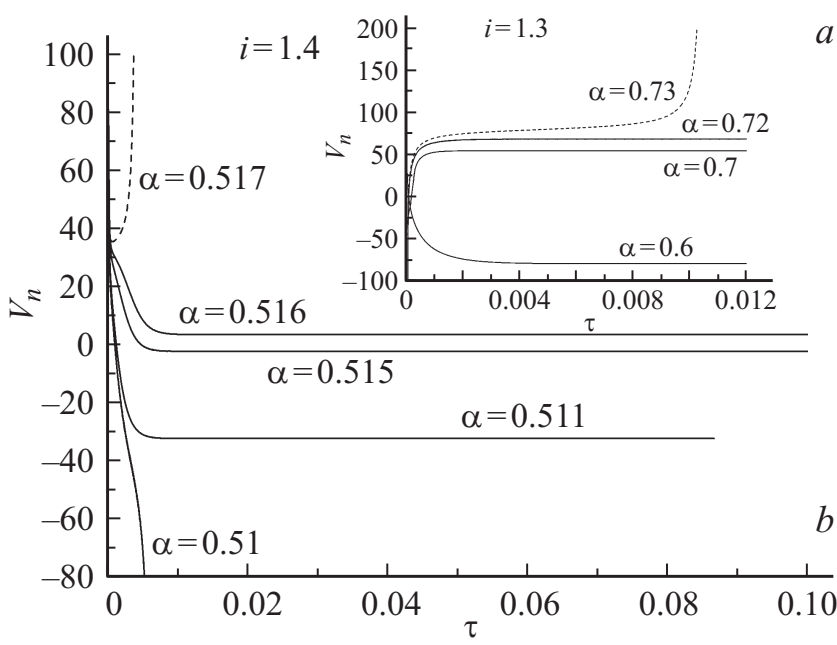

Рис. 7. Образование волны переключения при закритических токах и различных значениях параметра тепловой стабилизации (-- - - срыв токового режима).

$w(\theta)$ и $g(\theta)$ во всем диапазоне изменения температуры композита. В качестве иллюстрации сказанного на рис. 8 изображено изменение теплового состояния „хорошо“ стабилизированного композита $(\alpha=0.45)$ при действии температурного возмущения с параметрами $X_{1}=10$ и $\theta_{1}=8$ и закритическом токе $i=1.5$, превышающем в рассматриваемом случае ток полной стабилизации $i_{f}=1.4678$. Видно, что композит достаточно быстро теряет сверхпроводящие свойства, достигая равновесную температуру $\theta_{m}=1.0125$, несмотря на локальный характер исходного возмущения. В результате при $i>i_{f}$ однородные распределения температуры и напряженности электрического поля устанавливаются практически без изменения начальной длины возмущения.
На рис. 9 и 10 между собой сопоставлены результаты расчетов критических энергий для наиболее опасных локальных возмущений [8] и скоростей волны переключения как функции тока, полученных при $X_{1}=1$ в рамках обеих моделей для различных значений параметра тепловой стабилизации. Сплошные линии соответствуют расчетам, выполненным по модели со степенной ВАХ, штрих - по модели скачкообразного $S-N$-перехода. При минимальном токе распространения нормальной зоны $\varepsilon_{q} \rightarrow \infty$ (рис. 9), а установившиеся значения скорости тепловой неустойчивости равны нулю (рис. 8). При токе срыва критические энергии равны нулю, а скорости волны переключения - максимальны. Вертикальная прямая при $i=1$ на рис. 10 показывает границу полной тепловой стабилизации, которая следует из условия Стекли. Как отмечалось выше, параметры полной тепловой стабилизации для рассматриваемого композита со степенной ВАХ равны $\alpha_{f}=0.4642$, $i_{f}=1.4678$, т. е. для рассматриваемого композита диа-

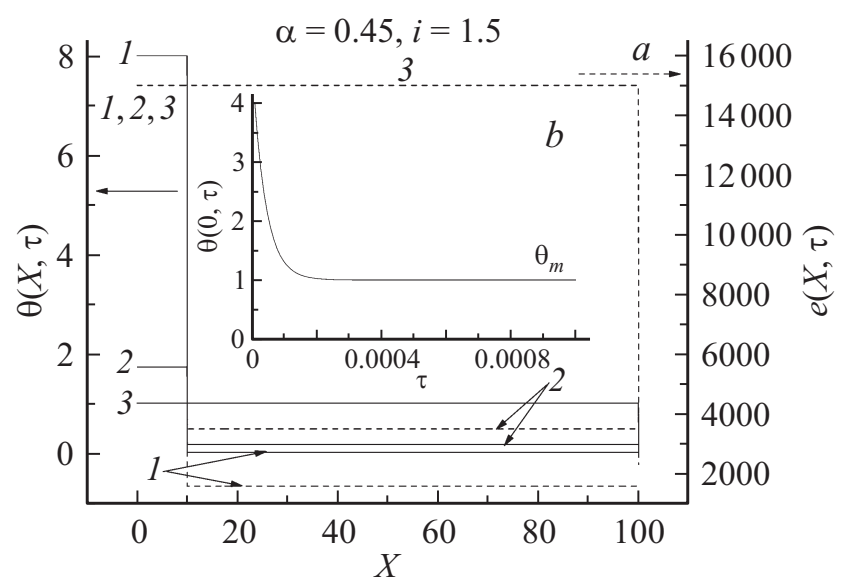

Рис. 8. Разрушение сверхпроводящих свойств композита при $i>i_{f}$ : - - распределение температуры, --- - распределение напряженности электрического поля.

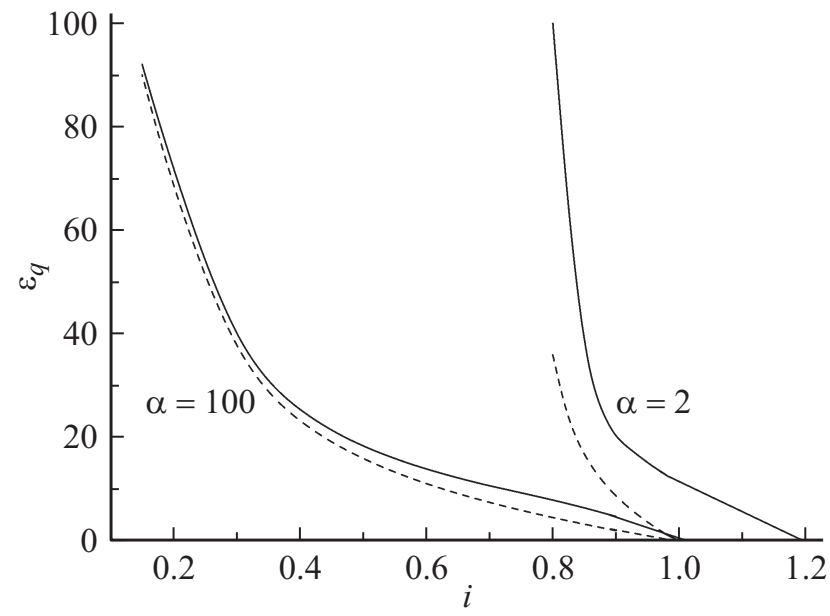

Рис. 9. Зависимость критических энергий от тока при локальном температурном возмущении и различных значениях параметра тепловой стабилизации. 


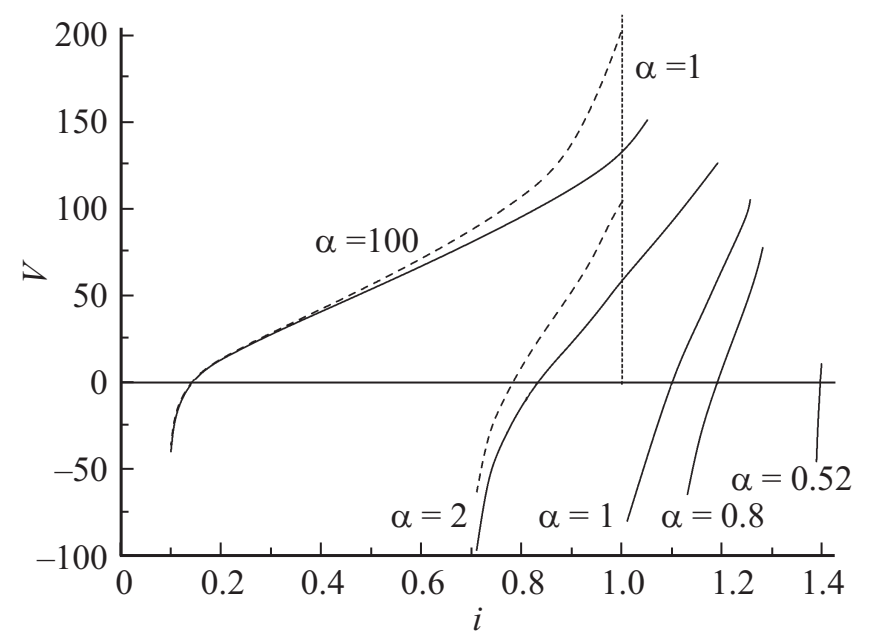

Рис. 10. Скорости распространения тепловой неустойчивости как функции тока при различных значениях параметра тепловой стабилизации.

пазон допустимых токов почти на 50\% больше, чем это следует из модели скачкообразного $S-N$-перехода. В целом представленные кривые показывают, что различие между моделями тем выше, чем ниже значение параметра тепловой стабилизации или чем ближе ток к условно заданному критическому значению. Наиболее заметное отличие имеет место при расчете скоростей распространения тепловых неустойчивостей даже несмотря на то, что минимальные токи существования нормальной зоны, как отмечалось выше, не отличаются друг от друга. В результате в модели скачкообразного $S-N$-перехода скорости тепловых неустойчивостей принимают максимальные значения независимо от параметра тепловой стабилизации при $i=1$. В то же время у технических сверхпроводников с реальной BAX значение тока, при котором скорости тепловых неустойчивостей максимальны, зависят от $\alpha$, а именно предельное значение тока увеличивается с уменьшением $\alpha$. Тем самым рис. 9, 10 наглядно демонстрируют, что учет закритических токовых режимов существенно расширяет диапазон тепловой стабильности технических сверхпроводников.

В заключение отметим, что выше для описания ВАХ сверхпроводника использовалось степенное уравнение, которое характерно для сверхпроводящих сред с множественными дефектами кристаллической решетки. Наряду со степенным уравнением реальные $E(J)$ характеристики могут описываться экспоненциальными уравнениями [9,13], если сверхпроводники имеют точечные дефекты. В этом случае ВАХ сверхпроводника следует из модели Кима-Андерсона, предполагая линейную зависимость потенциального барьера от тока. Однако, как было показано в [15], заметное отличие между теплоэлектродинамическими состояниями сверхпроводников со степенным и экспоненциальным уравнениями ВАХ будет возникать в случаях, когда $n<10$. В то же время технологии производства современных проводов на основе высокотемпературных сверхпроводников позволяют получать композиты, у которых показатели нарастания BAX имеют более высокие значения. Поэтому обсужденные выше условия тепловой стабилизации сверхпроводников со степенной ВАХ носят общий характер, которые определяют особенности тепловой стабилизации технических сверхпроводников с непрерывно нарастающей BAX.

\section{Выводы}

Предполагая, что плотность критического тока сверхпроводника линейно уменьшается с температурой, его BAX описывается степенным уравнением и теплоэлектрофизические параметры не зависят от температуры, выполнен анализ устойчивости сверхпроводящего состояния композитных сверхпроводников к температурным возмущениям. Он продемонстрировал, что условия их тепловой стабилизации не только количественно отличаются от соответствующих условий, следующих из существующей теории, основанной на скачкообразном $S-N$-переходе, но и имеют место качественные различия. Строго доказано, что критический ток композита, вводимый на основе скачкообразной аппроксимации его перехода из сверхпроводящего состояния в нормальное, не соответствует предельно допустимому значению тока, который может устойчиво протекать по композитному сверхпроводнику. Его реальное значение может быть как больше, так и меньше этой фиктивной величины в зависимости от параметра тепловой стабилизации.

Показано, что имеют место более оптимистичные условия устойчивости сверхпроводящего состояния по отношению к внешним тепловым возмущениям. В то же время расчетные значения скоростей необратимого распространения тепловой неустойчивости вдоль композита, определенные с учетом непрерывного нарастания BAX, меньше соответствующих значений, вычисленных в рамках модели скачкообразного $S-N$-перехода. Причем как при докритических, так и закритических токовых режимах тепловая неустойчивость распространяется вдоль композита в виде теплоэлектрической волны переключения.

Сформулировано условие полной тепловой стабилизации технических сверхпроводников, учитывающее нелинейный характер нарастания их ВАХ. Оно значительно расширяет диапазон токов, устойчивых к внешним тепловым возмущениям при интенсивном охлаждении. Поэтому известное условие Стекли $(\alpha=1)$, согласно которому сверхпроводящий композит полностью стабилен к тепловым возмущениям с произвольной энергией, не позволяет корректно определить условия, обеспечивающие полную тепловую стабилизацию композитов с реальными ВАХ. 


\section{Список литературы}

[1] Альтов В.А., Зенкевич В.Б., Кремлев М.Г., Сычев В.В. Стабилизация сверхпроводящих магнитных систем. М.: Энергоатомиздат, 1984. $312 \mathrm{c.}$

[2] Уилсон М. Сверхпроводящие магниты. М.: Мир, 1985. $407 \mathrm{c}$.

[3] Гуревич А.Вл., Мини, Р.Г., Рахманов А.Л. Физика композитных сверхпроводников. М.: Наука, 1987. 240 с.

[4] Altov V.V., Kremlev M.G. et al. // Cryogenics. 1978. Vol. 13. N 5. P. $420-422$.

[5] Chen W.Y., Purcell J.R. // J. Appl. Phys. 1978. Vol. 49. N 6. P. 3546-3553.

[6] Schmidt C. // Cryogenics. 1978. Vol. 18. N 10. P. 605-610.

[7] Anashkin O.P., Keilin V.E., Lyikov V.V. // Cryogenics. 1979. Vol. 19. N 2. P. $77-80$.

[8] Keilin V.E., Romanovsky V.R. // Cryogenics. 1982. Vol. 22. N 6. P. 313-317.

[9] Клименко Е.Ю., Мартовецкий Н.Н., Новиков С.И. В кн.: Техническая сверхпроводимость в электроэнергетике и электротехнике. М.: СЭВ, 1986. С. 161-187.

[10] Buznikov N.A., Pukhov A.A. // Cryogenics. 1996. Vol. 36. N 7. P. $547-553$.

[11] Lim H., Iwasa Y. // Cryogenics. 1997. Vol. 37. N 12. P. 789799.

[12] Романовский B.P. // Известия АН СССР. Энергетика и транспорт. 1986. № 1. C. 104-109.

[13] Polak M., Hlasnik I., Krempasky L. // Cryogenics. 1973. Vol. 13. N 12. P. 702-711.

[14] Romanovskii V.R., Watanabe K., Ken-ichiro Takahashi, Nishijima G., Awaji S. // Physica C. 2004. Vol. 416. N 34. P. $126-136$.

[15] Романовский В.Р. // ЖТФ. 2000. Т. 70. Вып. 12. С. 47-57. 